\title{
PROPAGACIÓN VEGETATIVA DE Retrophyllum rospigliosii (Pilg.) C.N. Page “ULCUMANO" EN CÁMARA DE SUBIRRIGACIÓN EN CHANCHAMAYO / PERÚ
}

\author{
VEGETATIVE PROPAGATION OF Retrophyllum rospigliosii (Pilg.) C.N. Page \\ “ULCUMANO” IN SUB-IRRIGATION CHAMBER IN CHANCHAMAYO/PERÚ
}

\author{
Pablo More ${ }^{1}$, José Cuellar ${ }^{2}$ y Evelin Salazar ${ }^{3}$
}

\begin{abstract}
Resumen
El ulcumano (Retrophyllum rospigliosii) es una importante conífera nativa del Perú, que actualmente está desapareciendo de las zonas donde era dominante debido a su excesivo aprovechamiento y problemas en su regeneración. Además, hay limitada información sobre su propagación vegetativa. Es por ello, que el objetivo de la investigación fue determinar la eficiencia de la propagación vegetativa del ulcumano en cámara de subirrigación. Para ello, se usaron cuatro concentraciones de AIB (0, 1000,3000 y 5000 ppm), dos edades de plantas madre (dos y ocho años) y dos tipos de estacas (apical y media); bajo un diseño factorial completamente al azar. Los resultados indican que, al término de las 17 semanas, el tratamiento T7 (correspondiente a estacas de tipo media, provenientes de plantas madres de dos años y tratadas con $3000 \mathrm{ppm}$ de AIB) obtuvo el mayor enraizamiento $(40 \%)$, longitud de raíces $(7 \mathrm{~mm})$ y número de raíces por estaca (2.2). La auxina AIB no influyó en el enraizamiento. Los tipos de estacas influyeron en la supervivencia, enraizamiento y brotación. La interacción entre la planta madre y tipo de estaca influyeron de manera significativa en el éxito del enraizamiento; las plantas madres de dos años y estacas tipo medio obtuvieron mayor supervivencia $(76.85 \%)$, enraizamiento $(32.5 \%)$ y brotación $(70 \%)$. Se concluye que es posible propagar el ulcumano usando estacas medias de árboles de dos años.
\end{abstract}

Palabras clave: propagación, enraizamiento, Retrophyllum rospigliosii, Podocarpaceae, auxina, fisiología.

\begin{abstract}
The ulcumano (Retrophyllum rospigliosii) is an important native conifer of Peru, which is currently disappearing from the areas where it was dominant, due to its excessive use and problems in its regeneration. Also, there is limited information on its vegetative propagation. For this reason, the objective was to determine the efficiency of the vegetative propagation of the ulcumano in the sub-irrigation chamber. Four concentrations of IBA were used $(0,1000,3000$ and 5000 ppm), two ages of mother plants (two and eight years) and two types of cuttings (apical and middle); under a completely random factorial design. The results indicate that, at the end of 17 weeks, the T7 treatment (corresponding to medium type cuttings, from two-year-old mother plants and treated with 3000 ppm IBA) obtained the highest rooting (40\%), root length $(7 \mathrm{~mm})$ and number of roots per cutting (2.2). Auxin AIB didn't influence rooting. Type cuttings influenced survival, rooting, and sprouting. The interaction between the cuttings mother plant and type of s had a significant influence on rooting success, two-year-old trees and medium-type cuttings obtained higher survival (76.85\%), rooting $(32.5 \%)$ and sprouting $(70 \%)$. It is concluded that it is possible to propagate ulcumano using middle cuttings of two-year-old trees.
\end{abstract}

Key words: propagation, rooting, Retrophyllum rospigliosii, Podocarpaceae, auxin, physiology.

\section{Introducción}

El Retrophyllum rospigliosii (Pilg.) C.N. Page "ulcumano" es una importante conífera nativa de la familia Podocarpaceae, que se distribuye en los bosques montanos tropicales del Perú, entre los 1200 a 3750 msnm (Mill, 2016). Se caracteriza por la excelente calidad de madera, fuste recto, copa pequeña la cual le permite obtener mayor cantidad de trozas aprovechables y se adapta muy bien a los sistemas agroforestales, en especial con el café (Coffea arabica). En el área donde se distribuye cumple una gran variedad de servicios ecológicos y ambientales, como la contribución a la firmeza del suelo, conservación de la humedad, regulación hídrica, protección de la biodiversidad y captura de carbono (Loján, 2003; Balvanera, 2012).

Según Gardner \& Thomas (2013), en el Perú existe evidencia que, muchos de los lugares, en donde se encontraban grandes extensiones naturales de esta especie, están desapareciendo o se han reducido considerablemente, estimándose la pérdida total de su área de ocupación en un $30 \%$; es por ello, que se ha catalogado como vulnerable, condición a la que también contribuye su excesivo aprovechamiento, y los problemas de regeneración. Se trata de una especie dioica, de semillas recalcitrantes y que son atacadas por larvas de coleópteros en el mismo árbol antes de ser diseminadas; asimismo, es susceptible a los cambios 
Enero - Julio 2021

supra anuales del clima (Cueva et al., 2013; Cueva \& Trujillo, 2016).

La clonación de árboles forestales, a través de estacas, es un método práctico, económico y viable para ser replicado y adaptado fácilmente en diferentes lugares, logrando mejorar la producción y conservación, especialmente en aquellas especies que presentan dificultades para su propagación sexual (Leakey, 2014; Jannat et al., 2017; Azad et al., 2018). Esta técnica ofrece la oportunidad de producir un suministro confiable y adecuado material de plantaciones con características superiores, de manera local, oportuna y rápida (Baul et al., 2010). Además, es un medio poderoso para capturar y explotar las ganancias genéticas de las mejores características fenotípicas y genotípicas en los programas de mejoramiento y multiplicación de árboles (Negash \& van Staden, 2003; Azad et al., 2018). Leakey (2014) y Azad et al. (2018) enfatizaron en el gran potencial de la domesticación de especies forestales tropicales a través de la propagación vegetativa.

Según Ragonezi et al. (2010), la mayoría de las especies forestales se pueden propagar por esta técnica; sin embargo, la capacidad de enraizamiento es muy específica para cada especie, en la que influyen diversos factores como la edad de la planta madre, tipo de estaca, tamaño y posición de las estacas, el medio en que se propaga, el tipo de regulador de crecimiento y su concentración, y las condiciones ambientales, por lo que se debe tener en cuenta a la hora de realizar un protocolo de propagación vegetativa (Leakey, 2014; Azad et al., 2018).

El ulcumano, a pesar de su valor, ha sido poco estudiado. Existe limitada información acerca de su ecología y comportamiento, así como de los métodos óptimos de manejo y propagación vegetativa. Investigaciones realizadas en Sudamérica y Perú muestran resultados poco satisfactorios de su propagación vegetativa (Cueva et al., 2013; Mill, 2016). Por este motivo, la presente investigación tuvo como objetivo determinar la eficiencia de tratamientos de propagación vegetativa, particularmente el efecto de los factores dosis de ácido indolbutírico (AIB), edad de la planta madre y tipo de estaca, sobre la capacidad de enraizamiento de Retrophyllum rospigliosii (Pilg.) C.N. Page "ulcumano" en cámara de subirrigación. Como hipótesis se planteó que alguna de las combinaciones de los factores antes mencionados mejoraría el enraizamiento de estacas del ulcumano.

\section{Materiales y métodos}

Ubicación del estudio y tiempo de ejecución

Los ensayos de enraizamiento se efectuaron en el Anexo San Ramón de la Estación Experimental Agraria Pichanaki (EEA Pichanaki) del Instituto Nacional de Innovación Agraria (INIA) en el Distrito de San Ramón, Provincia Chanchamayo, Región Junín, Perú, cuyas coordenadas UTM son: zona $18 \mathrm{~L}$,
8770394.70 m N y 461734.88 m E; a una elevación de $823 \mathrm{msnm}$. El estudio duró 120 días en condiciones de cámara de subirrigación.

El propagador de subirrigación

El propagador se construyó de acuerdo al diseño propuesto por Leakey et al. (1990) (Figura 1). El sustrato (arena gruesa) se desinfectó hirviéndolo por dos horas. Asimismo, se agregó 801 de agua a la cámara de subirrigación con el objetivo de mantener siempre húmedo el sustrato por efecto de capilaridad. Además, estuvo protegido por un techo de calamina y los costados por malla Raschel.

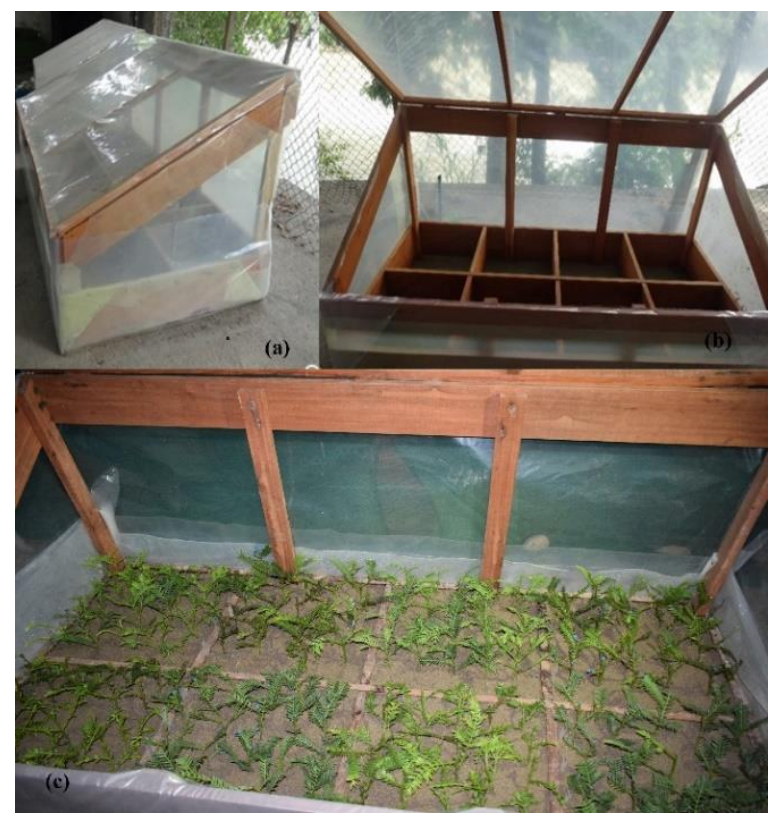

Figura 1. Cámara de subirrigación: exterior (a), interior (b) y con las estacas ulcumano instaladas (c).

\section{Proceso Experimental}

Las estacas se colectaron desde dos lugares. Del primero, vivero del Anexo San Ramón, se obtuvieron brotes de plantas de dos años de edad; las plantas se cortaron a $50 \mathrm{~cm}$ de altura para inducir la formación de brotes. Del segundo, fundo Mantus, Distrito de Vitoc, Chanchamayo, Junín, cuyas coordenadas UTM son 8755579.44 m S y 460560.90 m E, a una elevación de 1288 msnm, se obtuvieron de árboles de ocho años; las estacas se colectaron de brotes del fuste de árboles procedentes de plantaciones reproducidas por semillas.

La colecta y preparación de las estacas se efectuaron en horas de la mañana, seleccionando árboles sin presencia de plagas y/o enfermedades, de buenas características fenotípicas (fuste recto, ramas delgadas, ángulo de ramificación recto, sin bifurcación, copa cónica). Se colectaron 180 brotes del fuste, de cada brote se sacaron dos tipos de estacas (apical y media) con dimensiones $15-20 \mathrm{~cm}$ de longitud y $0.5-1 \mathrm{~cm}$ de diámetro. Una vez dimensionadas, las estacas se desinfectaron mediante inmersión por 10 minutos en una solución fungicida (Benomyl a 0.3\%); 
posteriormente fueron dispuestas en una mesa para un oreado de 20 minutos antes de la aplicación hormonal.

Para el tratamiento hormonal, se diluyó ácido indol3-butírico (AIB) químicamente puro (Caisson) en alcohol de $96^{\circ}$ para obtener soluciones con concentraciones de 1 000, 3000 y 5000 ppm de AIB. El testigo o control consistió en agua destilada. El método de aplicación de la hormona a las estacas fue por inmersión rápida, después de la cual se instalaron en la cámara del propagador.

Las condiciones de cultivo en el propagador fueron: temperatura promedio a las 8:00 am de $20.2^{\circ} \mathrm{C}$, a las $12: 00 \mathrm{~m}$ de $30.4{ }^{\circ} \mathrm{C}$ y a las $3: 00 \mathrm{pm}$ de $29.1{ }^{\circ} \mathrm{C}$; la humedad relativa promedio en la mañana fue $85 \%$, mientras que a las 12:00 m y 3:00 pm disminuyó a 54.7 y $53 \%$, respectivamente. Cuando el sustrato empezaba a secarse y el nivel del agua de la cámara se reducía, se procedía a regar.

Indicadores de respuesta

PORCENTAJE DE SUPERVIVENCIA $(\%)$ : Se contó el número de estacas que sobrevivieron al final de la investigación y se expresó como porcentaje sobre el total de unidades experimentales por tratamiento y repetición.

PORCENTAJE DE ENRAIZAMIENTO (\%): Porcentaje de estacas que lograron enraizar respecto al total de estacas por tratamiento y repetición. Se consideró enraizada a aquellas estacas con presencia de raíz mayor a $2 \mathrm{~mm}$ de longitud.

PORCENTAJE DE BROTACIÓN (\%): Porcentaje de estacas con brotes aéreos respecto al total de unidades experimentales por tratamiento y repetición. Se consideró como brotada a aquellas estacas con formación foliosa mayor a $1 \mathrm{~mm}$ de longitud.

NúMERO DE RAÍCES $\left(\mathrm{N}^{\circ}\right)$ : Número promedio de raíces principales por estaca por cada tratamiento y repetición.

LONGITUD DE RAÍZ: Longitud promedio de las raíces principales de las estacas de cada tratamiento y repetición que llegaron a enraizar en los 120 días.

NÚMERO DE BROTES $\left(\mathrm{N}^{\circ}\right)$ : Número promedio de brotes por estacas por tratamiento y repetición.

LONGITUD DE BROTES: Longitud promedio de los brotes de las estacas de cada tratamiento y repetición (brote $>1 \mathrm{~mm}$ de longitud).

\section{Diseño y análisis estadístico}

Se utilizó un diseño experimental completamente al azar con 16 tratamientos de estructura factorial $4 \times 2 \times 2$, siendo el factor $\mathrm{C}$ cuatro concentraciones de AIB ( 0 , 1000,3000 y 5000 ppm), el factor $\mathrm{P}$ dos edades de las plantas madre (dos y ocho años), y el factor E dos tipos de estaca (apical y media) (Tabla 1). Cada tratamiento contó con cuatro repeticiones y cinco estacas por unidad experimental, sumando en total 320 estacas como muestra.

Los valores de porcentaje fueron transformados mediante la fórmula del arcoseno de la raíz cuadrada del valor porcentual. A partir de los resultados, se realizó la prueba de normalidad, homocedasticidad de los datos y luego fueron sometidos al análisis de varianza y a la prueba de Tukey $(\mathrm{p}<0.05)$ con el software estadístico InfoStat con el fin de probar la existencia de diferencia significativa entre los tratamientos y los factores.

Tabla 1. Tratamientos de investigación para la propagación vegetativa de Retrophyllum rospigliosii.

\begin{tabular}{|c|c|c|c|}
\hline 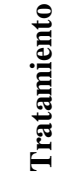 & Planta madre & $\begin{array}{l}\text { Tipo de } \\
\text { estaca }\end{array}$ & $\begin{array}{l}\text { Concentración } \\
\text { de AIB (ppm) }\end{array}$ \\
\hline $\mathrm{T} 1$ & A. 2 años & EA & 0 \\
\hline $\mathrm{T} 2$ & A. 2 años & EA & 1000 \\
\hline $\mathrm{T} 3$ & A. 2 años & EA & 3000 \\
\hline $\mathrm{T} 4$ & A. 2 años & EA & 5000 \\
\hline T5 & A. 2 años & EM & 0 \\
\hline T6 & A. 2 años & EM & 1000 \\
\hline $\mathrm{T} 7$ & A. 2 años & EM & 3000 \\
\hline $\mathrm{T} 8$ & A. 2 años & EM & 5000 \\
\hline T9 & A. 8 años & EA & 0 \\
\hline $\mathrm{T} 10$ & A. 8 años & EA & 1000 \\
\hline $\mathrm{T} 11$ & A. 8 años & EA & 3000 \\
\hline $\mathrm{T} 12$ & A. 8 años & EA & 5000 \\
\hline $\mathrm{T} 13$ & A. 8 años & EM & 0 \\
\hline T14 & A. 8 años & EM & 1000 \\
\hline $\mathrm{T} 15$ & A. 8 años & EM & 3000 \\
\hline T16 & A. 8 años & EM & 5000 \\
\hline
\end{tabular}

A. 2 años: árboles de 2 años de edad; A. 8 años: árboles de 8 años de edad; EA: estaca apical; EM: estaca media.

\section{Resultados}

Efecto de los tratamientos

En la Tabla 2 se detalla la existencia de las diferencias estadísticamente significativas entre los tratamientos $(\mathrm{p}=<0.0001)$. En términos absolutos, la mayor supervivencia se obtuvo con los tratamientos T9 y T5 (85\%), aunque estadísticamente no se diferencian de los restantes tratamientos, excepto de T1 y T16 que exhiben las supervivencias menores (25 y 30\%, respectivamente). El enraizamiento fue mayor en el T7 (40\%), pero no se diferencia de los tratamientos que exhiben enraizamientos iguales o superiores a $23 \%$ (T5-T8, T12-T14); en tanto que, los tratamientos T1T4, T9 y T10 no enrizaron o lo hicieron solo en un 5\%. Se detectó una relación directamente proporcional entre las variables supervivencia y enraizamiento $\left(\mathrm{R}_{\mathrm{AJ}}^{2}=0.26, \mathrm{p}=<0.0001\right)$. La formación de brotes fue mejor en el T5 (80\%), sin observarse relaciones claras con la supervivencia ni el enraizamiento. En términos de número y longitud de raíces y brotes promedios destacan con mejor desempeño los tratamientos $\mathrm{T} 7 \mathrm{y}$ $\mathrm{T} 8$, los que estuvieron conformados por estacas de tipo medio y de árboles de dos años, pero con diferentes concentraciones de AIB; sin embargo, en el T7 se usó la menor concentración de AIB (3 000 ppm). 
Enero - Julio 2021

Efecto de las concentraciones de AIB

Las dosis de AIB ensayadas no tuvieron efecto significativo en la supervivencia, enraizamiento, brotación, número y longitud de raíces principales y brotes en las estacas (Tablas 3 y 4); sin embargo, se observó una relación directamente proporcional, pero no estadísticamente significativa, entre dosis de AIB y porcentaje de enraizamiento.

Efecto de la edad de la planta madre

La edad de la planta madre no tuvo efecto significativo en la supervivencia y capacidad de enraizamiento. La brotación fue significativamente mayor en estacas de plantas madres de dos años, y hubo diferencia significativa (Tabla 3) en el número y longitud de raíces y brotes por estaca, siendo las plantas madres de dos años las que presentaron el mayor número $(1.06 \pm 0.2)$ y longitud $(2.54 \pm 0.6 \mathrm{~mm})$ de raíces promedio por estaca (Tabla 4). Solo se observó interacción significativa entre la edad de las plantas madres y la concentración de AIB (Tabla 3) para las variables supervivencia $(\mathrm{p}=0.0073)$ y longitud de brotes $(\mathrm{p}=0.0187)$. El mayor número de raíces $(1.5 \pm$ 0.2 ) se logró en estacas de plantas madres de dos años y con 5000 ppm de AIB (Figura 2a), en tanto que la longitud de raíces $(3.5 \pm 0.6 \mathrm{~mm})$ también fue mayor en estacas provenientes de plantas madres de dos años, pero tratadas con 3000 ppm de AIB (Figura 2b).

Efecto del tipo de estaca

El tipo de estaca influyó de manera significativa (Tabla 3) en todas las variables consideradas en este análisis (Tablas 3 y 4). Las estacas de tipo medio lograron un desempeño significativamente superior al de las estacas apicales en términos de porcentaje de supervivencia, enraizamiento y brotación, así como también en número y longitud de raíces y brotes. Según el análisis de varianza, la interacción entre los factores edad de la planta madre y tipo de estaca influyeron de manera significa $(\mathrm{p}=<0.0001)$ en el éxito de enraizamiento de estacas del ulcumano, y también sobre las demás variables evaluadas. Las estacas de tipo medio, provenientes de plantas madres de dos años, presentaron el mejor porcentaje de enraizamiento (32.5\%) (Figura 3a); lo mismo ocurrió con el porcentaje de brotación, donde se observó una alta diferencia estadística entre las estacas apicales (3.75\%) y medias $(70 \%)$ provenientes de plantas madres de dos años (Figura 3b). De igual forma, las estacas medias de plantas madres de dos años exhibieron mejor desempeño en el número $(2.1 \pm 0.2)$ y longitud $(5.1 \pm$ $0.4 \mathrm{~mm}$ ) de raíces (Figura 4), así como para el número $(1.9 \pm 0.2)$ y longitud $(4.4 \pm 0.4 \mathrm{~mm})$ de brotes por estaca. En la Tabla 5 se evidencia una relación positiva directa entre las variables número de raíces y número de brotes $\left(\mathrm{R}^{2}{ }_{\mathrm{AJ}}\right.$ : 0.53, $\left.\mathrm{p}=<0.0001\right)$, siendo las estacas de tipo medio procedentes de plantas madres de dos años las que exhibieron mayor formación de brotes en todo el proceso experimental, lo que al final del ensayo ha influido en mayor formación de raíces.
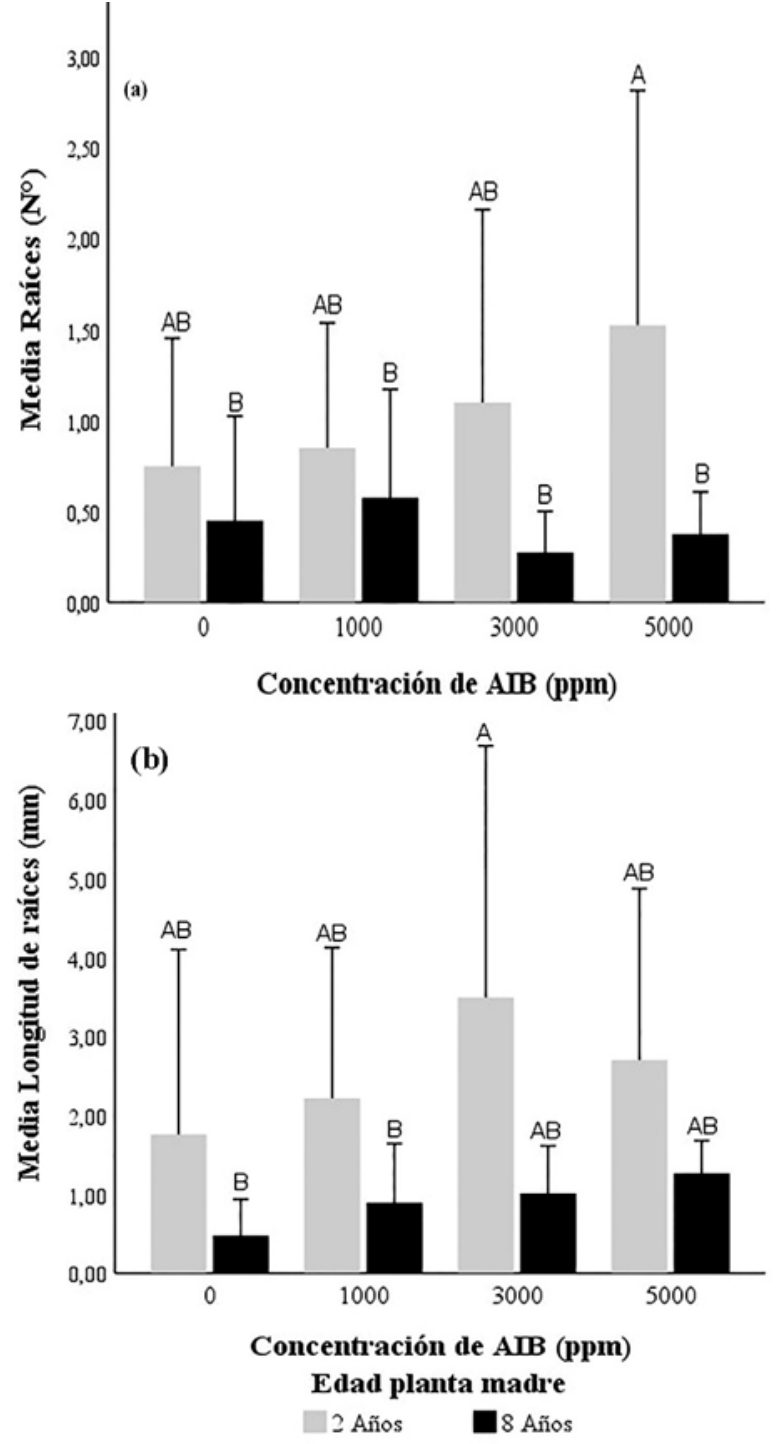

Figura 2. Número (a) y longitud (b) de raíces por estaca según concentraciones de AIB y edad del árbol madre. Valores medios \pm SE $(n=16)$ con letras diferentes presentaron diferencia significa según Tukey $(\mathrm{p}<0.05)$.

\section{Discusión \\ Eficiencia de tratamientos}

El tratamiento T7 fue el más eficiente, logrando hasta el $40 \%$ de enraizamiento, 75\% de brotación, 2.2 raíces por estaca y $7 \mathrm{~mm}$ de longitud promedio de sus raíces. Rivera et al. (2016) obtuvieron resultados similares con Pinus patula, logrando hasta un $60 \%$ de enraizamiento. Ojeda et al. (2019) no obtuvieron enraizamiento de ulcumano y solo llegaron a formar brotes y callos. Negash \& van Staden (2003) obtuvieron el $80 \%$ de enrizamiento con Podocarpusfalcatus, procedentes [las estacas] de plantas madres de tres meses y dos años, dando a conocer la importancia que cumple el factor edad de la planta madre en los tratamientos de la propagación vegetativa del ulcumano. 
Con estos resultados se puede demostrar que el ulcumano se puede propagar mediante estacas, tal como también afirman Cueva et al. (2013) y Mill (2016), pero su proceso de enraizamiento es muy lento, y así lo confirman Negash \& van Staden (2003) con otra especie de la familia Podocarpaceae.
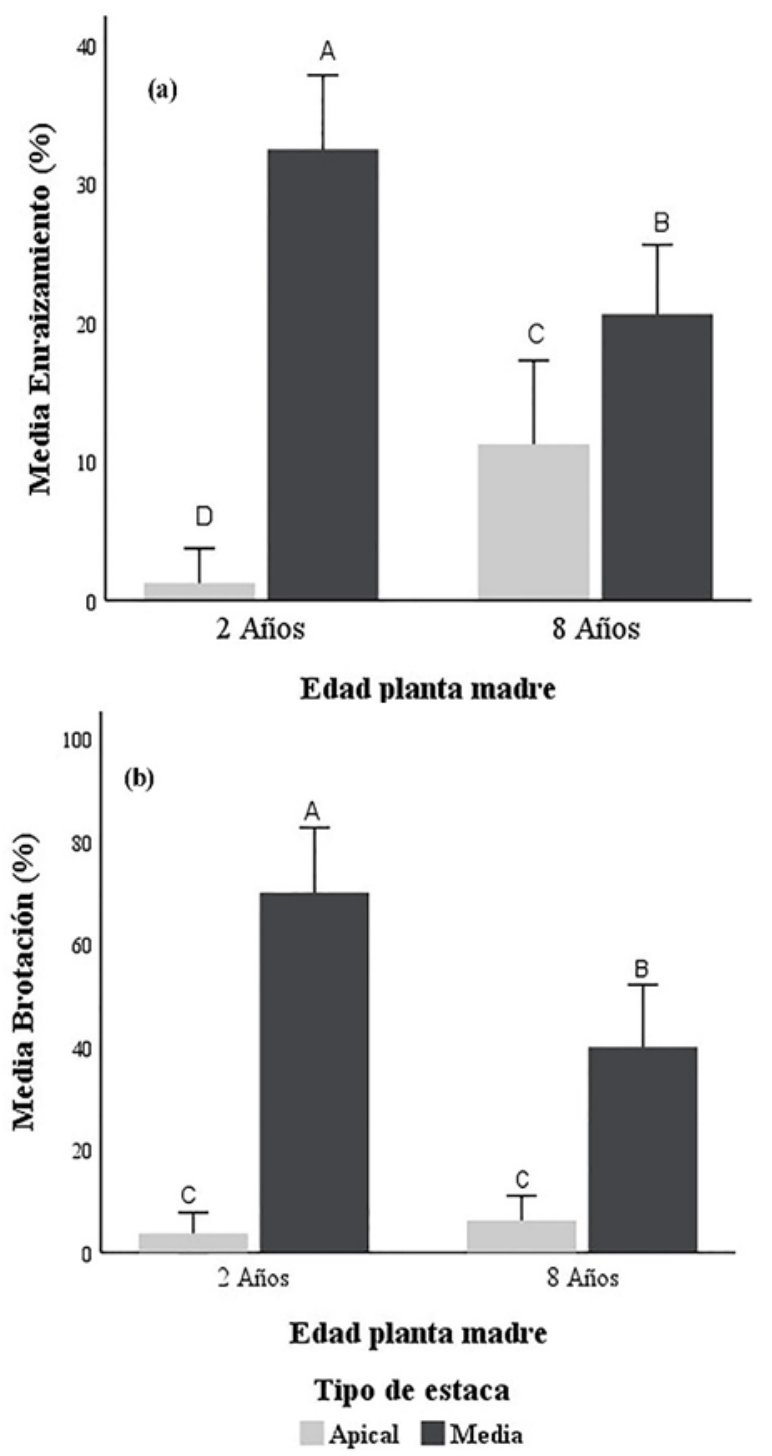

Figura 3. Enraizamiento (a) y brotación (b), según edad del árbol y el tipo de estaca. Valores medios \pm SE $(\mathrm{n}=16)$ con letras diferentes presentaron diferencia significa según Tukey $(\mathrm{p}<0.05)$.

\section{Efecto de las concentraciones de AIB}

Las dosis de AIB consideradas en el experimento no han tenido un efecto en el enraizamiento de estacas de ulcumano. Castillo et al. (2007) y Ojeda et al. (2019) tampoco encontraron efecto significativo de la auxina en el enraizamiento, logrando mayor éxito sin la aplicación de reguladores de crecimiento en especies de la familia Podocarpaceae. Sin embargo, numerosos estudios mencionan un efecto positivo del AIB sobre la rizogénesis aventicia; por ejemplo, P.falcatus respondió favorablemente entre 2000 y 8000 ppm de AIB (Negash \& van Staden, 2003). Al respecto, varios investigadores han confirmado que la concentración óptima de AIB varía de una especie a otra y se ve afectada por el pretratamiento de la planta madre (nutrición, luminosidad, radiación solar, poda); también influyen el tamaño y diámetro basal de los cortes, posición de los cortes en las plantas y número de hojas en los esquejes (Negash \& van Staden, 2003; Leakey, 2014). En efecto, la formación de raíces adventicias es un proceso de desarrollo sincronizado que implica varios eventos bioquímicos, fisiológicos e histológicos en la inducción, iniciación y elongación de la raíz (Negash \& van Staden, 2003).

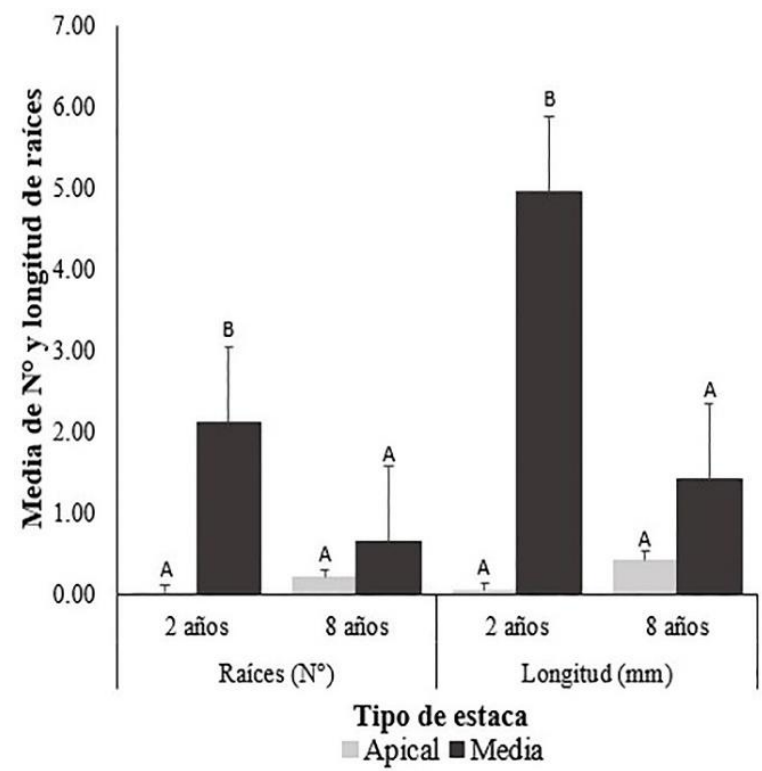

Figura 4. Número y longitud de raíces según edad del árbol y el tipo de estaca. Valores medios \pm SE $(n=16)$ con letras diferentes presentaron diferencia significa según Tukey $(\mathrm{p}<0.05)$.

Las concentraciones de AIB usadas no lograron generar un efecto significativo, pero sí se observó un ligero aumento de enrizamiento a medida que aumentó su concentración, por lo que para futuras investigaciones se pueden usar concentraciones más altas que las probadas en este trabajo, con el fin de perfeccionar y aumentar el porcentaje de enraizamiento de las estacas del ulcumano.

Efecto de la planta madre

Muchos estudios con árboles coníferos han demostrado que la propagación vegetativa por estacas en árboles maduros ha sido bastante difícil, ya que la edad del árbol afecta la competencia reproductiva y la tasa de crecimiento (Ragonezi et al., 2010); por ello, este factor cumple un papel importante a la hora de establecer protocolos de propagación.

La supervivencia de las estacas del ulcumano no se vio afectada por la edad del árbol donante, no obstante que el enraizamiento toma un largo tiempo durante el 
cual las estacas están expuestas a factores externos, como deshidratación y ataque de hongos, y se tiende a producir naturalmente una disminución de la supervivencia. Por lo mismo, es de importancia crucial controlar las condiciones ambientales y sanitarias durante el enraizamiento, de lo contrario, se pueden enfrentar a situaciones como las expuestas por Castillo et al. (2007), quienes obtuvieron muy bajos porcentajes de supervivencia (17 a 33\%) para dos especies de la familia Podocarpaceae.

Los resultados sugieren que el porcentaje de enraizamiento de estacas provenientes de plantas madres de 2 y 8 años no varía significativamente, pero disminuye el promedio de número y longitud de raíces conforme aumenta la edad de la planta madre, características principales para que las estacas sobrevivan a un futuro trasplante y, por ende, se puede testificar que los mejores resultados se han obtenido en árboles madre de dos años. Otros estudios indican valores de enraizamiento que disminuyen con la edad de la fuente de estacas. En efecto, Marín (1998) usó estacas de Podocarpus oleifolius de dos a tres años y obtuvo 74\% enraizamiento, mientras que Castillo et al. (2007) con árboles adultos de P. oleifolius, lograron enraizamientos de solo $2.4 \%$. Con estacas de Podocarpus falcatus de tres meses y dos años, Negash \& van Staden (2003) lograron porcentajes de enraizamiento superiores a los obtenidos con estacas de árboles de cuatro y ocho años de edad.

La reducción de la capacidad rizogénica asociada a la edad de la fuente de estacas posee diversas explicaciones, entre ellas, que los tejidos adultos son menos sensibles a las sustancias endógenas, disminución de la producción de auxinas con la edad, producción de inhibidores de enraizamiento o niveles fenólicos disminuidos que actúan como cofactores de auxinas (Hartmann \& Kester, 1988). Leakey (2014) sostiene que la pobre capacidad de enraizamiento de los brotes "maduros" puede atribuirse al "envejecimiento fisiológico" más que al "envejecimiento ontogénico". Del mismo modo, Wendling et al., (2014) sostienen que el envejecimiento ontogénico puede ser visible fenotípicamente, y a menudo se correlaciona con las características físicas, tales como tamaño de la planta, número de hojas y la capacidad para florecer. Conforme al envejecimiento ontogénico se puede indicar que los árboles de ocho años en campo ya habían presentado formación de flores el año anterior $y$, por lo mencionado anteriormente, en estos árboles a esta edad ya habría ocurrido el cambio de fase o que el árbol ya ingresó en proceso de maduración, dando como resultado la disminución de la capacidad de enraizamiento en árboles de ocho años, lo que ratifica que estos cambios se manifiestan a temprana edad en esta especie.

Efecto del tipo de estaca

El enraizamiento en la familia Podocarpaceae (Podocarpus oleifolius y Prumnopitys montana) muestra una tendencia a aumentar el enraizamiento desde las estacas apicales hacia las medias y basales (Castillo et al., 2007; Yépez, 2016). En este estudio no se ensayaron estacas basales, pero efectivamente en ulcumano las estacas medias enraizaron mejor que las apicales. Resultados similares obtuvieron Ruiz \& Mesén (2010) con Plukenetia volubilis. Esta tendencia posiblemente se asocia con las reservas de carbohidratos. Al respecto, Veierskov (1988) menciona que existe una relación positiva del contenido de carbohidratos con la capacidad rizogénica y con el número de raíces formadas en estacas de diferentes especies. Solo en algunas especies, las estacas apicales muestran buena respuesta por la mayor presencia de sustancias promotoras de enraizamiento (originadas en el ápice) y a una diferente calidad anatómica de la estaca (Mesén, 1998; Leakey, 2014). Este patrón se ajusta a los resultados del comportamiento del tipo de estaca en la propagación vegetativa.

De acuerdo con la interacción existente entre la edad de la planta madre y el tipo de estaca, los mejores resultados se han logrado con estacas de tipo medio provenientes de plantas madres de dos años. Según Leakey (2014), el éxito del enraizamiento está directamente relacionado al uso de material vegetativo joven y se pierde a medida que las plantas perennes envejecen. También, el tipo de estaca tiene un efecto significativo, como lo menciona Mesén (1998); estacas medias y basales de diferentes especies forestales han respondido mejor que las apicales debido a que estas últimas pueden ser muy herbáceas o tener menor reserva de carbohidratos.

Ruiz \& Mesén (2010) sostienen que la formación de brotes puede ser perjudicial en el enraizamiento de diferentes especies forestales; sin embargo, según estos resultados, las estacas de tipo medio procedentes de árboles de dos años han logrado el mayor número de raíces y brotes, habiendo similares resultados para Abies religiosa (Castillo et al., 2013).

En este estudio se demuestra que el ulcumano tiene un enraizamiento lento (120 días) y que el mejor material para propagar son estacas de tipo medio provenientes de árboles juveniles, descartándose el uso de estacas apicales de árboles menores a dos años, porque no resisten al proceso de enraizamiento.

\section{Conclusiones}

El mejor enraizamiento para estacas del ulcumano (Retrophyllum rospigliosii) se obtuvo con el T7, con $40 \%$ de enraizamiento, 2.2. raíces por estaca y con longitud de raíces principales de $7.0 \pm 1.9 \mathrm{~mm}$.

Los principales factores que influyeron en la propagación vegetativa fueron las estacas de tipo medio y procedentes de árboles de dos años en cámara de subirrigación.

La auxina AIB no influyó en el enraizamiento, pero se sugiere utilizar concentraciones más elevadas de las probadas. También es aconsejable probar ensayos más 
grandes (mayor número de estacas) con el uso de estacas basales, las que podrían tener mejor enraizamiento que las estacas medias y apicales.

El proceso de enraizamiento puede involucrar un periodo mayor al considerado en este ensayo (120 días), por cuanto al momento de la evaluación se encontraron estacas en proceso inicial de enraizamiento.

\section{Agradecimientos}

Agradecimiento especial al Instituto Nacional de Innovación Agraria (INIA, Perú) y al Proyecto Ulcumano 122_PI del Programa Nacional de Innovación Agraria (Perú) por el apoyo y el financiamiento para que sea posible esta investigación.

\section{Literatura citada}

Azad M.S., Alam M.J., Mollick A.S. \& Khan M.N.I. 2018. Rooting of cuttings of the wild Indian almond tree (Sterculia foetida) enhanced by the application of indole3-butyric acid (IBA) under leafy and non-leafy conditions. Rhizosphere 5: 8-15.

DOI: 10.1016/j.rhisph.2017.11.001.

Balvanera P. 2012. Los servicios ecosistémicos que ofrecen los bosques tropicales. Revista Ecosistemas, 21(1-2): 136-147.

https://www.revistaecosistemas.net/index.php/ecosistem as/article/view/33.

Baul T.K., Mezbahuddin M., Hossain M.M. \& Mohiuddin M. 2010. Vegetative propagation of Litsea monopetala, a wild tropical medicinal plant: Effects of indole-3-butyric acid (IBA) on stem cuttings. Journal of Forestry Research, 12(4): 228-235. DOI: $10.1007 / \mathrm{s} 11632-010-0409-3$.

Castillo J.D., López M.A., López J., Cetina V.M. \& Hernández T. 2013. Factores de influencia en el enraizamiento de Abies religiosa (Kunth) Schltdl. et Cham. Revista Chapingo - Serie Ciencias Forestales y del Ambiente, 19(1): 175-184. https://doi.org/10.5154/r.rchscfa.2011.06.038.

Castillo M., Cueva D., Aguirre N. \& Günter S. 2007. Propagación vegetativa de dos especies de la familia Podocarpaceae. Bosques Latitud Cero, 3: 3-5. https://www.rncalliance.org/WebRoot/rncalliance/Shops /rncalliance/4C15/95BB/5E58/166A/61A2/C0A8/D218/ 6FAA/Castillo_et_al_2007_propagacion_podocarpus.pd f.

Cueva N. \& Trujillo E. 2016. Biología reproductiva del pino Retrophyllum rospigliosii (Pilg.) C. N. Page. Cueva N. (Ed.). Colegio Integrado Nacional Oriente de Caldas. Pensilvania / Colombia.

Cueva N.C., Vélez D., Barrios A. \& Nieto V. 2013. Pino romerón [Retrophyllum rospigliosii (Pilger) C. N. Page], especie nativa potencial para la reforestación en zonas altoandinas de Colombia. Bogotá, Colombia. Corporación Nacional de Investigación y Fomento Forestal (CONIF®), Ministerio de Agricultura y Desarrollo Rural (MADR), Colegio Integrado Nacional Oriente de Caldas (CINOC).

Gardner M. \& Thomas P. 2013. Retrophyllum rospigliosii. The IUCN Red List of Threatened Species 2013: e.T34110A2846471. https://dx.doi.org/10.2305/IUCN.UK.20131.RLTS.T34110A2846471.
Hartmann H. \& Kester D. 1988. Propagación de plantas. Principios y prácticas. 5ta ed. Editorial Continental. México.

Jannat M., Hossain M.K., Alam M.S., Hossain M.A. \& Kamruzzaman M. 2017. Vegetative propagation of Tali (Palaquium polyanthum Engl.) for commercial harvest and landscape conservation. Rhizosphere, 3(1): 9-12. DOI: 10.1016/j.rhisph.2016.11.003.

Leakey R., Mesén J.F., Tchoundjeu Z., Longman K.A., Dick J.M., Matin A., Grace J., Munro R.C. \& Muthoka P.N. 1990. Low-Technology Techniques for the Vegetative Propagation of Tropical Trees. Commonwealth Forestry Review, 69(3): 247-257. https://www.jstor.org/stable/43738151.

Leakey R.R.B. 2014. Plant cloning: Macropropagation. In: Encyclopedia of Agriculture and Food Systems, 4: 349-359. https://doi.org/10.1016/B978-0-444-52512-3.00223-0.

Loján L. 2003. El verdor de los Andes Ecuatorianos: realidades y promesas. Proyecto Desarrollo Forestal Participativo en los Andes. Cámara Ecuatoriana del Libro - Núcleo de Pichincha. Ecuador.

Marín A. 1998. Propagación vegetativa de dos podocarpaceas del Bosque andino colombiano con problemas de propagación sexual. Smurfit. Carton, (186): 1-11.

Mesén F. 1998. Enraizamiento de estacas juveniles de especies forestales: uso de propagadores de Subirrigación. Manual técnico No 30 . Costa Rica.

Mill R.R. 2016. A Monographic Revision of Retrophyllum (Podocarpaceae). Edinburgh Journal of Botany, 73(2): 171-261. DOI: 10.1017/S0960428616000081.

Negash L. \& van Staden J. 2003. Vegetative propagation of the threatened East African yellowwood (Podocarpus falcatus). South African Journal of Botany, 69(2): 170-175. https://doi.org/10.1016/S0254-6299(15)30342-2.

Ojeda Q.E., Manayay M. \& Vergara S.E. 2019. Propagación por estacas de Retrophyllum rospigliosii Pilger y Tabebuia chrysantha (Jacq.) g. Nicholson con diferentes niveles de regulador de crecimiento, Jaén, Cajamarca, 2019. Revista Científica UNTRM - Ciencias Naturales e Ingeniería, 2(2), 33-38. http://dx.doi.org/10.25127/ucni.v2i2.515.

Ragonezi C., Klimaszewska K., Castro M.R., Lima M., de Olivera P. \& Zavattieri M.A. 2010. Adventitious rooting of conifers: influence of physical and chemical factors. Trees, 24(6): 975-992.

DOI: $10.1007 / \mathrm{s} 00468-010-0488-8$.

Rivera-Rodríguez M.O., Vargas-Hernández J.J., LópezUpton J., Villegas-Monter Á. \& Jiménez-Casas M. 2016. Enraizamiento de estacas de Pinus patula. Revista Fitotecnia Mexicana, 39(4): 385-392. http://www.revistafitotecniamexicana.org/documentos/3 9-4/5a.pdf.

Ruiz H. \& Mesén F. 2010. Efecto del ácido indolbutírico y tipos de estaquilla en el enraizamiento de sacha inchi (Plukenetia volubilis L.). Agronomía Costarricense, 34(2): 259-267. https://doi.org/10.15517/rac.v34i2.3636.

Veierskov B. 1988. Relations between carbohydrates and adventitious root formation. In: Davis T.D., Haissig B.E. \& Sankhla N. (Eds.) Adventitious root formation in cuttings. Advances in Plant Sciences Series. Volume 2. 70-78. Dioscorides Press. Portland, USA. 


\section{Enero - Julio 2021}

Wendling I., Trueman S.J. \& Xavier A. 2014. Maturation and related aspects in clonal forestry-Part I: Concepts, regulation and consequences of phase change. New Forests, 45(4): 449-471.

DOI: $10.1007 / \mathrm{s} 11056-014-9421-0$

Tabla 2. Valores promedios \pm SE del porcentaje de supervivencia, enraizamiento, brotación, $\mathrm{N}^{\mathrm{o}}$ y longitud de raíces y brotes por tratamiento en la propagación vegetativa de Retrophyllum rospigliosii, después de 17 semanas de ser instalados en cámaras de subirrigación.

\begin{tabular}{|c|c|c|c|c|c|c|c|}
\hline \multirow{2}{*}{ 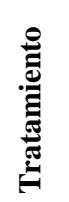 } & $\mathbf{S}$ & $\mathbf{E}$ & B & \multicolumn{2}{|c|}{ Raíces } & \multicolumn{2}{|c|}{ Brotes } \\
\hline & $\%$ & $\%$ & $\%$ & $\mathbf{N}^{\mathbf{o}}$ & mm & $\mathbf{N}^{0}$ & $\mathbf{m m}$ \\
\hline T1 & $25 \pm 9.6 \mathrm{c}$ & $0 \mathrm{e}$ & $0 \mathrm{~d}$ & $0.0 \mathrm{c}$ & $0.0 \mathrm{~d}$ & $0.0 \mathrm{~b}$ & $0.0 \mathrm{~d}$ \\
\hline $\mathrm{T} 2$ & $50 \pm 12.9 \mathrm{abc}$ & $5 \pm 5.0 \mathrm{de}$ & $5 \pm 5.0 \mathrm{~cd}$ & $0.1 \pm 0.1 \mathrm{c}$ & $0.1 \pm 0.1 \mathrm{~d}$ & $0.1 \pm 0.1 \mathrm{~b}$ & $0.1 \pm 0.1 \mathrm{~d}$ \\
\hline T3 & $50 \pm 10.0 \mathrm{abc}$ & $0 \mathrm{e}$ & $5 \pm 5.0 \mathrm{~cd}$ & $0.0 \mathrm{c}$ & $0.0 \mathrm{~d}$ & $0.1 \pm 0.1 \mathrm{~b}$ & $0.1 \pm 0.1 \mathrm{~d}$ \\
\hline $\mathrm{T} 4$ & $50 \pm 5.8 \mathrm{abc}$ & $0 \mathrm{e}$ & $5 \pm 5.0 \mathrm{~cd}$ & $0.0 \mathrm{c}$ & $0.0 \mathrm{~d}$ & $0.1 \pm 0.1 \mathrm{~b}$ & $1.1 \pm 1.1 \mathrm{~cd}$ \\
\hline T5 & $85 \pm 5.0 \mathrm{a}$ & $25 \pm 5.0 \mathrm{abcd}$ & $80 \pm 8.2 \mathrm{a}$ & $1.5 \pm 0.5 \mathrm{abc}$ & $3.5 \pm 2.1 \mathrm{abcd}$ & $1.8 \pm 0.4 \mathrm{ab}$ & $5.5 \pm 0.7 \mathrm{a}$ \\
\hline T6 & $65 \pm 5.0 \mathrm{ab}$ & $30 \pm 5.8 \mathrm{abc}$ & $50 \pm 17.3 \mathrm{abc}$ & $1.7 \pm 0.4 \mathrm{abc}$ & $4.3 \pm 1.1 \mathrm{abcd}$ & $1.2 \pm 0.6 \mathrm{ab}$ & $2.5 \pm 0.9 \mathrm{abcd}$ \\
\hline $\mathrm{T} 7$ & $75 \pm 5.0 \mathrm{a}$ & $40 \pm 4.1 \mathrm{a}$ & $75 \pm 5.0 \mathrm{a}$ & $2.2 \pm 0.7 \mathrm{ab}$ & $7.0 \pm 1.9 \mathrm{a}$ & $2.3 \pm 0.8 \mathrm{a}$ & $4.6 \pm 0.8 a b c$ \\
\hline $\mathrm{T} 8$ & $80 \pm 8.2 \mathrm{a}$ & $35 \pm 5.0 \mathrm{ab}$ & $75 \pm 9.6 \mathrm{a}$ & $3.1 \pm 0.6 \mathrm{a}$ & $5.4 \pm 0.8 \mathrm{ab}$ & $2.5 \pm 0.4 \mathrm{a}$ & $4.8 \pm 1.1 \mathrm{ab}$ \\
\hline T9 & $85 \pm 9.6 \mathrm{a}$ & $5 \pm 5 \mathrm{de}$ & $0 \mathrm{~d}$ & $0.1 \pm 0.1 \mathrm{c}$ & $0.1 \pm 0.1 \mathrm{~d}$ & $0.0 \mathrm{~b}$ & $0.0 \mathrm{~d}$ \\
\hline T10 & $60 \pm 0.0 a b c$ & $5 \pm 5 \mathrm{de}$ & $10 \pm 5.8 \mathrm{bcd}$ & $0.1 \pm 0.1 \mathrm{c}$ & $0.1 \pm 0.1 \mathrm{~d}$ & $0.1 \pm 0.1 \mathrm{~b}$ & $0.6 \pm 0.5 \mathrm{~d}$ \\
\hline T11 & $65 \pm 5.0 \mathrm{ab}$ & $10 \pm 5.8 \mathrm{cde}$ & $10 \pm 5.8 \mathrm{bcd}$ & $0.1 \pm 0.1 \mathrm{c}$ & $0.5 \pm 0.3 \mathrm{~cd}$ & $0.1 \pm 0.1 \mathrm{~b}$ & $0.6 \pm 0.4 \mathrm{~d}$ \\
\hline $\mathrm{T} 12$ & $70 \pm 5.8 \mathrm{a}$ & $25 \pm 2.9 \mathrm{abcd}$ & $5 \pm 5 \mathrm{~cd}$ & $0.6 \pm 0.2 \mathrm{bc}$ & $1.0 \pm 0.4 \mathrm{~cd}$ & $0.1 \pm 0.1 \mathrm{~b}$ & $0.2 \pm 0.2 \mathrm{~d}$ \\
\hline $\mathrm{T} 13$ & $70 \pm 5.8 \mathrm{a}$ & $30 \pm 5.8 \mathrm{abc}$ & $55 \pm 12.6 \mathrm{ab}$ & $0.9 \pm 0.5 \mathrm{bc}$ & $0.9 \pm 0.4 \mathrm{~cd}$ & $1.6 \pm 0.5 \mathrm{ab}$ & $2.1 \pm 0.4 \mathrm{abcd}$ \\
\hline T14 & $55 \pm 5 a b c$ & $23 \pm 4.8 \mathrm{abcd}$ & $45 \pm 15.0 \mathrm{abcd}$ & $1.1 \pm 0.5 \mathrm{bc}$ & $1.7 \pm 0.5 \mathrm{bcd}$ & $0.8 \pm 0.3 \mathrm{ab}$ & $3.3 \pm 1.4 \mathrm{abcd}$ \\
\hline $\mathrm{T} 15$ & $75 \pm 9.6 \mathrm{a}$ & $15 \pm 2.9$ bcde & $35 \pm 9.6 \mathrm{abcd}$ & $0.5 \pm 0.2 b c$ & $1.6 \pm 0.4 \mathrm{bcd}$ & $1.0 \pm 0.5 \mathrm{ab}$ & $1.5 \pm 0.8 \mathrm{bcd}$ \\
\hline T16 & $30 \pm 5.8 \mathrm{bc}$ & $15 \pm 2.9$ bcde & $25 \pm 9.6 \mathrm{bcd}$ & $0.2 \pm 0.0 \mathrm{c}$ & $1.5 \pm 0.2 \mathrm{bcd}$ & $0.8 \pm 0.3 \mathrm{ab}$ & $0.8 \pm 0.4 \mathrm{~d}$ \\
\hline$\overline{\mathrm{X}}$ & 61.9 & 16.6 & 30.0 & 0.7 & 1.7 & 0.8 & 1.7 \\
\hline
\end{tabular}

S: Supervivencia; E: enraizamiento; B: Brotación. Letras diferentes representan diferencia significativa según Tukey (p < 0.05)

Tabla 3. Análisis de varianza para los tratamientos en la propagación vegetativa de Retrophyllum rospigliosii "ulcumano", después de 17 semanas de ser instalados en cámaras de subirrigación.

\begin{tabular}{|c|c|c|c|c|c|c|c|}
\hline \multirow[t]{2}{*}{ Indicador } & \multirow{2}{*}{$\begin{array}{l}\mathbf{S} \\
\%\end{array}$} & \multirow{2}{*}{$\begin{array}{l}\mathbf{E} \\
\%\end{array}$} & \multirow{2}{*}{$\begin{array}{l}\text { B } \\
\%\end{array}$} & \multicolumn{2}{|c|}{ Raíces } & \multicolumn{2}{|c|}{ Brotes } \\
\hline & & & & $\mathbf{N}^{\circ}$ & $\mathbf{m m}$ & $\mathbf{N}^{o}$ & $\mathbf{m m}$ \\
\hline Tratamiento & $<0.0001 *$ & $<0.0001 *$ & $<0.0001^{*}$ & $<0.0001^{*}$ & $<0.0001^{*}$ & $<0.0001 *$ & $<0.0001^{*}$ \\
\hline $\mathrm{C}$ & 0.1448 & 0.6200 & 0.7223 & 0.5072 & & 0.5481 & \\
\hline $\mathrm{P}$ & & 0.6614 & $0.0043^{*}$ & $0.0004 *$ & $0.0002 *$ & $0.0174 *$ & $0.0012 *$ \\
\hline $\mathrm{E}$ & $0.0091 *$ & $<0.0001 *$ & $<0.0001 *$ & $<0.0001 *$ & $<0.0001 *$ & $<0.0001 *$ & $<0.0001 *$ \\
\hline $\mathrm{CxP}$ & $0.0073 *$ & 0.1587 & 0.2825 & 0.2040 & 0.6948 & 0.3816 & $0.0187 *$ \\
\hline $\mathrm{CxE}$ & $0.0473^{*}$ & 0.1941 & 0.1737 & 0.9759 & 0.4448 & 0.4133 & 0.4043 \\
\hline PxE & $<0.0001 *$ & $<0.0001 *$ & $0.0009 *$ & $<0.0001 *$ & $<0.0001 *$ & $0.0146 *$ & $0.0010 *$ \\
\hline
\end{tabular}

C: Concentración de AIB; P: Edad de planta madre; E: Tipo de estaca; S: Supervivencia; E: enraizamiento; B: Brotación. *Presentan diferencia significativa según Tukey $(\mathrm{p}<0.05)$. 
Tabla 4. Valores promedios \pm SE del porcentaje de supervivencia, enraizamiento, brotación, $\mathrm{N}^{\mathrm{o}}$ y longitud de raíces y brotes para los factores en la propagación vegetativa del Retrophyllum rospigliosii, después de 17 semanas de ser instalados en cámaras de subirrigación.

\begin{tabular}{|c|c|c|c|c|c|c|c|c|}
\hline \multirow[t]{2}{*}{ Factor } & & \multirow{2}{*}{$\begin{array}{l}\mathrm{S} \\
\%\end{array}$} & \multirow{2}{*}{$\begin{array}{l}\mathbf{E} \\
\%\end{array}$} & \multirow{2}{*}{$\begin{array}{l}\text { B } \\
\%\end{array}$} & \multicolumn{2}{|c|}{ Raíces } & \multicolumn{2}{|c|}{ Brotes } \\
\hline & & & & & $\mathrm{N}^{\mathbf{0}}$ & $\mathbf{m m}$ & $\mathbf{N}^{\mathbf{o}}$ & $\mathbf{m m}$ \\
\hline \multirow{4}{*}{$\begin{array}{l}\text { Concentración } \\
\text { de AIB (ppm) }\end{array}$} & 0 & $66.25 \pm 7.2 \mathrm{a}$ & $15.00 \pm 3.8 \mathrm{a}$ & $33.75 \pm 9.6 \mathrm{a}$ & $0.59 \pm 0.2 \mathrm{a}$ & $1.12 \pm 0.6 \mathrm{a}$ & $0.84 \pm 0.3 \mathrm{a}$ & $1.91 \pm 0.6 \mathrm{a}$ \\
\hline & 1000 & $57.50 \pm 3.9 \mathrm{a}$ & $15.63 \pm 3.6 \mathrm{a}$ & $27.50 \pm 7.5 \mathrm{a}$ & $0.71 \pm 0.2 \mathrm{a}$ & $1.56 \pm 0.5 \mathrm{a}$ & $0.55 \pm 0.2 \mathrm{a}$ & $1.60 \pm 0.5 \mathrm{a}$ \\
\hline & 3000 & $66.25 \pm 4.4 \mathrm{a}$ & $16.25 \pm 4.2 \mathrm{a}$ & $31.25 \pm 8.2 \mathrm{a}$ & $0.69 \pm 0.3 \mathrm{a}$ & $2.25 \pm 0.8 \mathrm{a}$ & $0.85 \pm 0.3 \mathrm{a}$ & $1.69 \pm 0.5 \mathrm{a}$ \\
\hline & 5000 & $57.50 \pm 5.7 \mathrm{a}$ & $18.75 \pm 3.6 a$ & $27.50 \pm 8.1 \mathrm{a}$ & $0.95 \pm 0.4 \mathrm{a}$ & $1.98 \pm 0.6 \mathrm{a}$ & $0.85 \pm 0.3 \mathrm{a}$ & $1.73 \pm 0.6 \mathrm{a}$ \\
\hline \multirow{2}{*}{$\begin{array}{l}\text { Edad planta } \\
\text { madre (años) }\end{array}$} & 2 & $60.00 \pm 4.2 \mathrm{a}$ & $16.88 \pm 3.2 \mathrm{a}$ & $36.88 \pm 6.8 b$ & $1.06 \pm 0.2 b$ & $2.54 \pm 0.6 \mathrm{~b}$ & $0.99 \pm 0.2 b$ & $2.32 \pm 0.5 \mathrm{~b}$ \\
\hline & 8 & $63.75 \pm 3.4 \mathrm{a}$ & $15.94 \pm 2.1 \mathrm{a}$ & $23.13 \pm 4.4 \mathrm{a}$ & $0.41 \pm 0.1 \mathrm{a}$ & $0.91 \pm 0.1 \mathrm{a}$ & $0.56 \pm 0.1 \mathrm{a}$ & $1.14 \pm 0.3 \mathrm{a}$ \\
\hline \multirow{2}{*}{ Tipo de estaca } & Apical & $56.88 \pm 3.9 b$ & $6.25 \pm 1.8 b$ & $5.00 \pm 1.6 b$ & $0.10 \pm 0.1 \mathrm{~b}$ & $0.22 \pm 0.1 \mathrm{~b}$ & $0.07 \pm 0.0 \mathrm{~b}$ & $0.31 \pm 0.6 \mathrm{~b}$ \\
\hline & Media & $66.88 \pm 3.5 \mathrm{a}$ & $26.56 \pm 2.1 \mathrm{a}$ & $55.00 \pm 5.1 \mathrm{a}$ & $1.37 \pm 0.2 \mathrm{a}$ & $3.23 \pm 0.5 \mathrm{a}$ & $1.48 \pm 0.2 \mathrm{a}$ & $3.15 \pm 0.4 \mathrm{a}$ \\
\hline
\end{tabular}

S: Supervivencia; E: Enraizamiento; B: Brotación. Letras diferentes representan diferencia significativa según Tukey $(\mathrm{p}<0.05)$.

Tabla 5. Matriz de correlación de Pearson y $\mathrm{R}^{2}$ ajustado para el porcentaje de supervivencia, enraizamiento, brotación, $\mathrm{N}^{\circ}$ y longitud de raíces y brotes en la propagación vegetativa del Retrophyllum rospigliosii, después de 17 semanas de ser instalados en cámaras de subirrigación.

\begin{tabular}{|c|c|c|c|c|c|c|c|c|c|}
\hline \multicolumn{3}{|c|}{ Variables de respuesta } & \multirow[t]{2}{*}{ S (\%) } & \multirow{2}{*}{$\begin{array}{l}\mathbf{E}(\%) \\
0.509^{*}\end{array}$} & \multirow{2}{*}{$\begin{array}{l}\mathbf{B}(\%) \\
0.519^{*}\end{array}$} & \multirow{2}{*}{$\begin{array}{l}\text { Raíces } \\
\left(\mathbf{N}^{\mathbf{o}}\right)\end{array}$} & \multirow{2}{*}{$\frac{(\mathbf{m m})}{0.400^{*}}$} & \multirow{2}{*}{$\begin{array}{l}\text { Brotes } \\
\left(\mathbf{N}^{\mathbf{o}}\right)\end{array}$} & \multirow{2}{*}{$\frac{(\mathbf{m m})}{0.478^{*}}$} \\
\hline S (\%) & & $\begin{array}{l}\text { Correlación } \\
\text { de Pearson }\end{array}$ & & & & & & & \\
\hline & & $\mathrm{R}^{2}$ ajustado & & 0.248 & 0.258 & 0.190 & 0.146 & 0.190 & 0.216 \\
\hline \multirow{2}{*}{\multicolumn{2}{|c|}{$\mathrm{E}(\%)$}} & $\begin{array}{l}\text { Correlación } \\
\text { de Pearson }\end{array}$ & $0.509^{*}$ & & $0.812^{*}$ & $0.802^{*}$ & $0.793^{*}$ & $0.740^{*}$ & $0.726^{*}$ \\
\hline & & $\mathrm{R}^{2}$ ajustado & 0.248 & & 0.654 & 0.638 & 0.622 & 0.541 & 0.52 \\
\hline \multirow{2}{*}{\multicolumn{2}{|c|}{ B (\%) }} & $\begin{array}{l}\text { Correlación } \\
\text { de Pearson }\end{array}$ & $0.519^{*}$ & $0.812^{*}$ & & $0.814^{*}$ & $0.775^{*}$ & $0.871^{*}$ & $0.905^{*}$ \\
\hline & & $\mathrm{R}^{2}$ ajustado & 0.258 & 0.654 & & 0.657 & 0.594 & 0.754 & 0.817 \\
\hline \multirow[t]{4}{*}{ Raíces } & $\left(\mathrm{N}^{\mathbf{o}}\right)$ & $\begin{array}{l}\text { Correlación } \\
\text { de Pearson }\end{array}$ & $0.450^{*}$ & $0.802^{*}$ & $0.814^{*}$ & & $0.890^{*}$ & $0.734^{*}$ & $0.806^{*}$ \\
\hline & & $\mathrm{R}^{2}$ ajustado & 0.19 & 0.638 & 0.657 & & 0.788 & 0.532 & 0.644 \\
\hline & $(\mathbf{m m})$ & $\begin{array}{l}\text { Correlación } \\
\text { de Pearson }\end{array}$ & $0.400^{*}$ & $0.793^{*}$ & $0.775^{*}$ & $0.890^{*}$ & & $0.703^{*}$ & $0.749^{*}$ \\
\hline & & $\mathrm{R}^{2}$ ajustado & 0.146 & 0.622 & 0.594 & 0.788 & & 0.486 & 0.553 \\
\hline \multirow[t]{4}{*}{ Brotes } & $\left(\mathbf{N}^{0}\right)$ & $\begin{array}{l}\text { Correlación } \\
\text { de Pearson }\end{array}$ & $0.450^{*}$ & $0.740^{*}$ & $0.871^{*}$ & $0.734^{*}$ & $0.703^{*}$ & & $0.727^{*}$ \\
\hline & & $\mathrm{R}^{2}$ ajustado & 0.19 & 0.541 & 0.754 & 0.532 & 0.486 & & 0.521 \\
\hline & $(\mathbf{m m})$ & $\begin{array}{l}\text { Correlación } \\
\text { de Pearson }\end{array}$ & $0.478^{*}$ & $0.726^{*}$ & $0.905^{*}$ & $0.806^{*}$ & $0.749^{*}$ & $0.727^{*}$ & \\
\hline & & $\mathrm{R}^{2}$ ajustado & 0.216 & 0.52 & 0.817 & 0.644 & 0.553 & 0.521 & \\
\hline
\end{tabular}

S: Supervivencia; E: Enraizamiento; B: Brotación. *La correlación es significativa en el nivel 0.05 (bilateral).

\footnotetext{
${ }^{1}$ Facultad de Ciencias Ambientales / Universidad Científica del Sur. Villa el Salvador, Lima / Perú. jmore@cientifica.edu.pe.

${ }^{2}$ Facultad de Ciencias Forestales / Universidad Nacional Agraria La Molina. Lima / Perú. eloycuellar@lamolina.edu.pe.

${ }^{3}$ Instituto Nacional de Innovación Agraria. La Molina / Lima / Perú. esalazar@inia.gob.pe.
} 